\title{
Proofs, proofs, proofs, and proofs ${ }^{\star}$
}

\author{
Manfred Kerber \\ Computer Science, University of Birmingham \\ Birmingham B15 2TT, England \\ http://www.cs.bham.ac.uk/ ${ }^{\sim m m k}$
}

\begin{abstract}
In logic there is a clear concept of what constitutes a proof and what not. A proof is essentially defined as a finite sequence of formulae which are either axioms or derived by proof rules from formulae earlier in the sequence. Sociologically, however, it is more difficult to say what should constitute a proof and what not. In this paper we will look at different forms of proofs and try to clarify the concept of proof in the wider meaning of the term. This has implications on how proofs should be represented formally.
\end{abstract}

\section{Introduction}

There is a relatively clear definition of what a proof is. The concept has been clarified in the last 150 years with the development of logic and in the last 50 to 60 years with the development of systems which formalize the results of these investigations in formal computer systems. Mathematicians, however, seem not to have much changed their view of proofs 11 Sure, they have some knowledge of the results in foundations but if they work in fields such as statistics, group theory, or geometry then the formalization of proof is only of marginal interest, although the concept of proof itself is at the core of the whole of mathematics.

Is this just a matter of ignorance? Or rather of professionalism? And what are the consequences for our field which tries to offer support for mathematicians?

In order to approach these questions an account of the development of the concept of proof in different fields is given. We first take a look at the development in logic (section 2) $)^{2}$. Next we see the consequences this had on working mathematicians and their attitude towards formal proofs (section 3). The development in logic has strongly influenced the development of deduction systems. In section 4 we take a brief look at deduction systems. Then some consequences for the field of mathematical knowledge management are discussed. Essentially we argue that the representation of proofs should be flexible enough to serve different purposes in order to be able to communicate proofs at different levels: checkable proofs, abstract high-level proofs, proof ideas/plans, and false proofs.

\footnotetext{
* The final publication of this paper is available at www.springerlink.com

${ }^{1}$ Obviously for those working on the foundations of mathematics this is different. The generalization 'mathematicians' does not mean all mathematicians, but most typical mathematicians.

${ }^{2}$ The claim is not that there is a single view in the different fields. Even a single person may have different views at different times or in different contexts.
} 


\section{The Logician's View}

In the second half of the 19th century and in the early 20th century, a rigorous definition of the concept of proof was given. Inspired by the rigorous work of Euclid, Hilbert axiomatized geometry and developed a programme to be carried through for all of mathematics. Whitehead and Russell wrote the Principia Mathematica [23] which started to implement the grand vision to reduce all of mathematics to logic. Logicians like Boole and Frege developed propositional and predicate logic, and Gentzen the calculus of Natural Deduction.

As Frege put it, the vision was ([7] quoted from [10, p.6f]):

In apprehending a scientific truth we pass, as a rule, through various degrees of certitude. Perhaps first conjectured on the basis of an insufficient number of particular cases, a general proposition comes to be more and more securely established by being connected with other truths through chains of inferences, whether consequences are derived from it that are confirmed in some other way or whether, conversely, it is seen to be a consequence of propositions already established. Hence we can inquire, on the one hand, how we have gradually arrived at a given proposition and, on the other, how we can finally provide it with the most secure foundation. The first question may have to be answered differently for different persons; the second is more definite, and the answer to it is connected with the inner nature of the propositions considered. The most reliable way of carrying out a proof, obviously, is to follow pure logic ... Everything necessary for a correct inference is expressed in full ... nothing is left to guesswork.

Hilbert's definition of proof as a sequence of formulae which are either axioms or generated by rules from elements coming earlier in the sequence is now quite standard in logic books. Natural Deduction calculi as introduced by Gentzen (see, e.g., [19]) are an extension of this definition. For instance, Andrews 2, p.11] defines strictly formally the notions of a proof (from hypotheses and then a proof of a well-formed formula (wff)). Then he defines "A theorem is a wff which has a proof."

On a more philosophical level there has been a dispute what should constitute a rigorous proof, since even with this clarification the question was not fully settled. Most notably there was a dispute between Hilbert and Brouwer on the right approach to mathematics, in which the question of constructive proofs versus 'classical' proofs played a major role. Hilbert wanted, in particular, defend the 'paradise' of (infinite) sets provided by Cantor, whereas Brouwer was wary about the concept of infinity and insisted on constructiveness. At the time, Brouwer's view was considered by many mathematicians as too restrictive (and probably is still by many today). With the advent of computers the idea of constructive proofs, however, gained great attraction since proving and programming became the same activity. For details of the dispute see [10]. There are other disputes, e.g., about the axiom of choice and about the rigour of diagrams in reasoning. For the argument here, it suffices to state that even in the rigorous area of logical 
foundations there can be dispute about what should constitute a proof and what not ${ }^{3}$

\section{The Mathematician's View}

Mathematicians seem to have ignored the development in formal logic to a large degree. The start of the rapid development of modern logic can be put roughly to the mid 19th century. However, the start of the rapid development of modern mathematics is about 200 years older 4

As Kline [12, p.256] notes, the "Bourbakists expressed their position on logic in an article in the Journal of Symbolic Logic (1949): 'In other words, logic, so far as we mathematicians are concerned, is no more and no less than the grammar of the language which we use, a language which had to exist before the grammar could be constructed.' Future developments in mathematics may call for modifications of the logic. This had happened with the introduction of infinite sets and,... it would happen again."

In a similar line, Bourbaki (4 quoted from [12, p.320]) doubts that one of the goals of logicians, to make mathematics free from contradictions, is feasible:

Historically speaking, it is of course quite untrue that mathematics is free from contradictions; non-contradiction appears as a goal to be achieved, not as a God-given quality that has been granted to us once for all. Since the earliest times, all critical revisions of the principles of mathematics as a whole, or of any branch in it, have almost invariably followed periods of uncertainty, where contradictions did appear and had to be resolved.... There are now twenty-five centuries during which the mathematicians have had the practice of correcting their errors and thereby seeing their science enriched, not impoverished; this gives them the right to view the future with serenity.

Theorems with their proofs are at the core of mathematics and play a significant role in the working of mathematicians. Hardy describes in $\S 12$ of [9] two examples of theorems (with proofs) which he calls 'first-rate': First the theorem that there are infinitely many prime numbers (the proof is indirect, assume that you have finitely many, multiply them all and add 1 . The new number is not divisible by any prime number, which gives a contradiction.) and second the theorem that $\sqrt{2}$ is irrational (again an indirect proof: assume $\sqrt{2}=a / b$ with $a$ and $b$ two integers which have no common divisor. Then $2 \cdot b^{2}=a^{2}$. It follows that $a^{2}$ and hence $a$ must be even, but then $b$ must be even as well, which gives a contradiction).

In $\S 18$ Hardy states then:

\footnotetext{
${ }^{3}$ It should be noted, however, that there is a clear language and it is very clear to the participants of a dispute what they are talking about.

4 And of course there are always precursors, Aristotle as the father of logic, Archimedes who was close to inventing calculus almost 2000 years before Newton and Leibniz.
} 
In both theorems (and in the theorems, of course, I include the proofs) there is a very high degree of unexpectedness, combined with inevitability and economy. The arguments take so odd and surprising a form; the weapons used seem so childishly simple when compared with the farreaching results; but there is no escape from the conclusions. There are no complications of detail - one line of attack is enough in each case; and this is true too of the proofs of many much more difficult theorems, the full appreciation of which demands quite a high degree of technical proficiency. We do not want many "variations" in the proof of a mathematical theorem: "enumeration of cases," indeed is one of the duller forms of mathematical argument. A mathematical proof should resemble a simple and clear-cut constellation, not a scattered cluster in the Milky Way.

Proofs often follow established patterns. Often they are invented, doubted by other, later generally accepted, and finally re-used, taught, and generally recognized. Examples are the $\epsilon-\delta$ criterion (to establish continuity), diagonalization (to establish the impossibility of certain properties, e.g. halting problem, incompleteness, uncountability), mathematical induction (to reason about infinite structures), infinitesimals (to reason about differentiation).

From a logicians point of view mathematical proofs are more like proof plans. This is reflected in the education of mathematics. Proof principles such as the $\epsilon-\delta$ criterion are taught in lectures, without a strictly formal treatment. Many of these principles are even taught in concrete proofs which have exemplary character and can be generalized later on to many other cases. Formal logic, however, is not necessarily part of the education of a mathematician. In consequence, the concept of a proof is much less strict, and 'mathematics is a MOTLEY of techniques of proof' as Wittgenstein put it [24, p. 176f] 5

This means that the concept of proof is not fixed once and for all but requires the possibility for extension. Practically, mathematicians treat proofs and proof methods as first class objects, that is, just as they introduce new concepts they may introduce new proof principles, describe them and then use them. For this reason mathematicians focus on their special fields of expertise and consider the study of logic as one field among others. And if this field is not their specialty and particular area of expertise then they do what professionals do with fields they consider only as marginally relevant: they give it only marginal attention.

\section{The Deductionist's View}

In formal communities such as the theorem proving community, the logicians' view of the concept of proof has (for good reasons) been predominant, but not been the only view. Davis distinguishes two communities, the logic oriented and the cognitive oriented communities.

\footnotetext{
${ }^{5}$ Still there is a general assumption in mathematics that in principle it is possible to extend these mathematical proofs (proof plans) to full logic level proofs if necessary.
} 


\section{Automated Theorem Proving}

As Davis [6, p.5] states:

With the ready availability of serious computer power, deductive reasoning, especially as embodied in mathematics, presented an ideal target for those interested in experiments with computer programs that purported to implement the "higher" human faculties. This was because mathematical reasoning combines objectivity with creativity in a way difficult to find in other domains. For this endeavor, two paths presented themselves. One was to understand what people do when they create proofs and write programs emulating that process. The other was to make use of the systematic work of the logicians reducing logical reasoning to standard canonical forms on which algorithms could be based.

Since the groundbreaking work in logic in the early 20th century was very close to implementation it led to the dream to build machines that can solve hard problems fully automatically. The invention of the resolution principle by Robinson [21] which made search spaces finitely branching was a great breakthrough and led to the possibility to prove many theorems fully automatically. In parallel there was a smaller community which was interested in the cognitive aspects of theorem proving (by Newell and others, followed up in the proof planning work by Bundy and others). At least motivationally the work is linked to psychological evidence [20] that deductive reasoning plays a very important role in human intelligence and that some proof rules like Modus ponens are universally accepted while others are accepted only by a minority. Related in this context is also the work on diagrammatic reasoning (see, e.g. 11] which shows that reasoning falsely considered for some time as inferior, can be made very precise.

In general, however, the dream of full automation has not come true at large. There are fascinating exceptions such as the proof of the Robbins problem ([15]), but still mathematicians do not have theorem proving machines on their desks which they use to a similar degree as they use typesetting programs or computer algebra systems. And possibly not everybody would want such a machine, since as Hardy put it in [9, § 10] "there is nothing in the world which pleases even famous men ... quite so much as to discover, or rediscover, a genuine mathematical theorem." and we can add "and a genuine mathematical proof." (but proofs are parts of the theorem for Hardy anyway.) Why leaving the fun to a machine?

There has been a different community at least since the 1960s, namely the community interested in being able to check proofs by a machine. On first sight this looks like a much less ambitious goal, but it turned out to be actually much more difficult than anticipated. We will look at this next.

\section{Proof Checking}

The perhaps two most prominent approaches to proof checking - from which other systems have been derived - are Automath [5] and Mizar [22. The goal is 
not to find proofs automatically but to check proofs. De Bruijn summarizes his dream in 1994 [16, p.210] as follows:

As a kind of dream I played (in 1968) with the idea of a future where every mathematician would have a machine on his desk, all for himself, on which he would write mathematics and which would verify his work. But, by lack of experience in such matters, I expected that such machines would be available in 5 years from then. But now, 23 years later, we are not that far yet.

In many ways this dream is more exciting, since firstly it looks more feasible and secondly it is something mathematicians and professionals working in related fields can appreciate more. Although proof checkers have been extended by useful extensions which allow for higher-level proofs, most notably by tactics which allow to reduce many steps in a proof to a single user interaction, even 16 years after de Bruijn's retrospective we are still not there and mathematicians do not widely use the corresponding systems. However, they can be used and some do use them, most notably there is the Flyspeck Project [8] in which Hales (and others) are formalizing his proof of the Kepler conjecture.

\section{$5 \quad$ How to Make Systems more Accepted?}

Systems which deal with proofs can be built for different purposes and different purposes result in different requirements. Only some of them are currently adequately supported by mathematical knowledge management systems. Let us look at the most common purposes/contexts in which proofs are communicated:

Education: In an educational context proofs will be presented and/or jointly developed in order to teach the concept. A teacher may want to teach how to find a proof but more typically will teach how to write up a proof so that it is of an acceptable standard. These are two different modes as Pólya 18, p. vi], pointed out:

We secure our mathematical knowledge by demonstrative reasoning, but we support our conjectures by plausible reasoning ... Demonstrative reasoning is safe, beyond controversy, and final. Plausible reasoning is hazardous, controversial, and provisional. ... In strict reasoning the principal thing is to distinguish a proof from a guess, a valid demonstration from an invalid attempt. In plausible reasoning the principal thing is to distinguish a guess from a guess, a more reasonable guess from a less reasonable guess. ... [plausible reasoning] is the kind of reasoning on which his [a mathematician's] creative work will depend.

Proof development: Here the scenario is that of a mathematician or a group of mathematicians developing a proof. They do not know yet the details of the proof (or even whether there is a proof), they may have some ideas which 
may be vague and informal. A blackboard and a piece of chalk seem to be the tools of choice and systems at best offer the functionality of a blackboard and chalk. In Pólya's words, the game is mainly about plausible reasoning at this stage. The task of providing support is particularly challenging since proof attempts, ideas, partial proof plans may have to be communicated.

Automation: If automation is the objective and proofs are found, automated theorem provers typically can document a formal proof object which can be independently checked. This object can be communicated.

Correctness: If correctness of arguments is sought then proofs must be checkable. At a calculus level the different formal systems implemented allow this. Human mathematicians, however, can check proofs at a less formal level. Support at this level is still patchy, although important steps have been made in an area which is labelled as the development of a mathematical vernacular (going back to de Bruijn and the Automath project, and continued by Nederpelt and Kamareddine [17]).

For any of the different activities there is the question: What kind of information is necessary and how should it be represented?

A proof is an argument that should convince the reader (interpreter) of the truth of a statement (certain axioms and assumptions given). That is, a proof is a relationship between the argument and the reader, and the reader has to come with some level of knowledge.

If we know a lot, then a proof can be more concise. If we know the theorem already then we do not need to be convinced. If we know little, then we need a detailed argument which convinces us beyond reasonable doubt (some may say beyond any doubt) of the correctness of the theorem. In this respect a proof is a proof only with respect to a receiver/reader. "Nothing can be explained to a stone, the reader must understand something beforehand." as McCarthy formulated it (1964, p.7), quoted from [1, p.8] and analogously we can state that "Nothing can be explained to God, since he understands everything beforehand." or as Ayer [3, p.85f] put it:

The power of logic and mathematics to surprise us depends, like their usefulness, on the limitations of our reason. A being whose intellect was infinitely powerful would take no interest in logic and mathematics. For he would be able to see at a glance everything that his definitions implied, and, accordingly could never learn anything from logical inference which he was not fully conscious of already. But our intellects are not of this order. It is only a minute proportion of the consequences of our definitions that we are able to detect at a glance. Even so simple a tautology as " $91 \times 79=7189$ " is beyond the scope of our immediate apprehension. To assure ourselves that " 7189 " is synonymous with " $91 \times 79$ " we have to resort to calculation, which is simply a process of tautological transformation - that is, a process by which we change the form of expression without altering their significance. The multiplication tables are rules for carrying out this process in arithmetic, just as the laws of logic are 
rules for the tautological transformation of sentences expressed in logical symbolism or in ordinary language.

Typically, we are in between the stone and God: We know certain theorems and proofs and are happy to accept certain arguments when they are mentioned in a new proof and others not. We can fill in certain gaps, but not others. We have intelligence which goes beyond checking substitutions and matching, which can convince us that a theorem is really a theorem. A proof should give us a good reason why we should not doubt the correctness of the theorem at an appropriate level. Going back to a logic level proof is typically like being dragged on a level on which we do not see the wood for the trees.

Indeed proofs come in various formats, they can be presented at different levels of abstraction and can be quite different in style and details. In order to represent and support them appropriately we need to know what they are needed for and have to reflect the purpose and the level of understanding and knowledge of the reader. The reader may know the proof already or know a similar proof (and would be quite quick at understanding the new one). The reader may have no intuition - possibly the statement is even counter intuitive - and would have to check steps slowly and carefully. Or the reader may not be able to understand the proof in a reasonable amount of time at all since they lack the corresponding knowledge and would require a significant course in a whole field of mathematics before they can appreciate the arguments 6

In a familiar area, mathematicians know which arguments to accept and where to be careful. They are well aware of fallacies to avoid, that is, we have positive and negative information at our disposal and avoid the fallacies as they are described by Maxwell in [14. Maxwell's examples deal, for instance, with non-apparent divisions by zero, with problems with integration by parts, and with incorrectly drawn auxiliary diagrams in geometric proofs. Maxwell distinguishes between fallacies, where things go wrong on a deeper level (and proof checking on a high-level may wrongly succeed) and howlers, where the incorrectness of the argument is apparent (and a wrong argument may still give the correct result).

That the mathematical notion of a proof is subject to change, not strictly formal, and not beyond doubt has most convincingly been described by Lakatos 13. in an analysis of the history of the Euler polyhedron theorem, which had an exciting history of proofs and subsequent counterexamples, which led to improved proofs and more sophisticated counterexamples. This has not led to a general distrust in proofs. Although the theorem is not central to mathematics, still as Hardy put it $[9, \S 12]$ - a mathematician offers the game, and a contradiction may cast doubt on the correctness of mathematics as a whole. However, the sequence of proof, counterexample, proof can be seen very much in the spirit of

\footnotetext{
${ }^{6}$ Obviously the borders are not sharp. We may know a similar proof, and actually we would not remember every single step. Having a good intuition, having some intuition, and having no intuition, or a counter intuition is again fluid. The proof of the Robbins problem was so hard for humans since they did not have an intuition of the Robbins algebras.
} 
the quote in section 3 of Bourbaki that "mathematicians have had the practice of correcting their errors and thereby seeing their science enriched, not impoverished."

Theorem proving and checking proofs is a social activity and in a highly specialized society there are different reasons why we believe a theorem and its proof. Only few will actually have the knowledge, the capacity, and the time to understand complicated proofs like that of the Fermat-Wiles theorem or the Kepler conjecture. Still most of us will accept that there are proofs and that the theorems hold. The two theorems mentioned by Hardy, however, have much simpler proofs and it belongs to the folklore to know their proofs.

We see that there is a broad spectrum of proofs. Typically natural language in combination with diagrams is used to store and communicate proofs. Some types of proof (formal logical proofs, some types of proof plans) can be represented in a format which is better suited to mechanical manipulation (e.g. to proof checking) than natural language. Other types are still difficult to formalize. Work on the mathematical vernacular is certainly useful in order to formalize the variety of proofs. An advanced approach to understand informal proofs at a linguistic level has been carried through by Zinn 25. He analyzes the linguistic structure of proofs and builds internal structures, which reflect the inner logic of the proofs. This opens a way to understanding and checking informal mathematical discourse.

\section{Summary}

Proofs come at different levels and with different intentions. They are written for readers/checkers who/which must have certain competences. A human mathematician who knows a theorem very well knows and can communicate proofs of it at different levels: the gist of it, which allows other experts to reconstruct a full proof, a proof plan for a less proficient reader/checker, and a low level proof for a checker with little information in the field. Likewise an expert can understand proofs on different levels.

A system that has deep knowledge about proofs would be able to link the different levels. Achieving such a human level of expertise looks AI-hard unfortunately. On the other hand this has its attraction as Davis states, since it "combines objectivity with creativity." (Generalized) proof plans can offer a framework which is general enough to capture the different levels. Linking different levels and understanding different levels simultaneously will remain a hard problem for some time, and proof will remain a colourful concept.

\section{References}

1. Philip S. Abrams. An APL machine. SLAC-114 UC-32 (MISC), Stanford University, Stanford, California, 1970.

2. Peter B. Andrews. An Introduction to Mathematical Logic and Type Theory: To Truth through Proof. Academic Press, Orlando, Florida, USA, 1986. 
3. Alfred Jules Ayer. Language, Truth and Logic. Victor Gollancz Ltd, London, United Kingdom, 2nd edition, 1951 edition, 1936.

4. Nicolas Bourbaki. Théorie des ensembles. Éléments de mathématique, Fascicule 1. Hermann, Paris, France, 1954.

5. Nicolaas Govert de Bruijn. A survey of the project Automath. In J.P. Seldin, J.R. Hindley, editors, To H.B. Curry - Essays on Combinatory Logic, Lambda Calculus and Formalism, pages 579-606. Academic Press, London, United Kingdom, 1980.

6. Martin Davis. The early history of automated deduction. In Alan Robinson and Andrei Voronkov, editors, Handbook of Automated Reasoning - Volume I, pages 5-14. Elsevier Science, Amsterdam, The Netherlands, 2001.

7. Gottlieb Frege. Begriffsschrift, eine der arithmetischen nachgebildete Formelsprache des reinen Denkens. Halle, 1879.

8. Thomas Hales. The Flyspek Project. http://code.google.com/flyspeck/, 2010.

9. Godfrey Hardy. A Mathematician's A pology. Cambridge University Press, London, United Kingdom, 1940.

10. Jean van Heijenoort, editor. From Frege to Gödel - A Source Book in Mathematical Logic, 1879-1931. Harvard Univ. Press, Cambridge, Massachusetts, USA, 1967.

11. Mateja Jamnik. Mathematical Reasoning with Diagrams: From Intuitions to Automation. CSLI Press, Stanford, California, USA, 2001.

12. Morris Kline. Mathematics - The Loss of Certainty. Oxford University Press, New York, USA, 1980.

13. Imre Lakatos. Proofs and Refutations. Cambridge University Press, 1976.

14. E.A. Maxwell. Fallacies in Mathematics. Cambridge University Press, Cambridge, United Kingdom, 1959.

15. William McCune. Solution of the Robbins problems. Journal of Automated Reasoning, 19(3):263-276, 1997. See also http://www.mcs.anl.gov/home/mccune/ar/robbins/

16. Rob Nederpelt, Herman Geuvers, and Roel de Vrijer, editors. Selected Papers on Automath, volume 133 of Studies in Logic and the Foundations of Mathematics. North-Holland, Amsterdam, The Netherlands, 1994.

17. Rob Nederpelt and Fairouz Kamareddine. An abstract syntax for a formal language of mathematics. In The Fourth International Tbilisi Symposium on Language, Logic and Computation, 2001. http://www. cedar-forest.org/forest/papers/conference-publications/tbilisi01.ps

18. George Pólya. Mathematics and Plausible Reasoning. Princeton University Press, Princeton, New Jersey, USA, 1954. Two volumes, Vol. 1: Induction and Analogy in Mathematics, Vol. 2: Patterns of Plausible Inference.

19. Dag Prawitz. Natural Deduction - A Proof Theoretical Study. Almqvist \& Wiksell, Stockholm, Sweden, 1965.

20. Lance J. Rips. The Psychology of Proof - Deductive Reasoning in Human Thinking. The MIT Press, Cambridge, Massachusetts, USA, 1994.

21. John Alan Robinson. A machine oriented logic based on the resolution principle. Journal of the ACM, 12:23-41, 1965.

22. Andrzej Trybulec. The Mizar logic information language. Studies in Logic, Grammar and Rhetoric, 1, 1980. Białystok, Poland.

23. Alfred North Whitehead and Bertrand Russell. Principia Mathematica, volume I. Cambridge University Press, Cambridge, United Kingdom; 1910.

24. Ludwig Wittgenstein. Bemerkungen über die Grundlagen der Mathematik, volume 506. Suhrkamp-Taschenbuch Wissenschaft, Frankfurt, Germany; 3rd edition, 1989.

25. Claus Zinn. Understanding Informal Mathematical Discourse. $\mathrm{PhD}$ thesis, Friedrich-Alexander-Universität Erlangen-Nürnberg, Erlangen, Germany, 2004. 